\title{
El agua en L'Autre Alceste de Alfred Jarry
}

\author{
Alexandrescu, Ioana \\ Universidad Autónoma de Barcelona, Universidad de Oradea, Ioana.Alexandrescu@uab.cat
}

\section{Resumen}

En una de sus excéntricas ocurrencias, Alfred Jarry aseguraba que el agua es un veneno corrosivo, capaz de destrozar, con una sola gota, la pureza de otro líquido. Lo mismo le decía a su amiga, la escritora Rachilde, advirtiéndole sobre el peligro de envenenarse, puesto que el agua contiene todos los microbios «de la tierra y del cielo». En este contexto vital claramente reacio a considerar el agua como una bebida adecuada, surgieron escritos que la valorizan, por otra parte, en su amplia y ancestral capacidad simbólica. La obra de Alfred Jarry comprende múltiples declinaciones del imaginario acuático, aún poco exploradas por la crítica. Nuestro trabajo se propone analizar las particularidades de la presencia acuática en uno de sus escritos más abundantes en este aspecto, L’Autre Alceste.

Palabras clave: Alfred Jarry; L'Autre Alceste; Agua

\section{Résumé}

Dans l'un de ses propos excentriques, Alfred Jarry affirmait que l'eau est un poison corrosif, capable de détruire, par une seule goutte, la pureté d'un autre liquide. C'est ce qu'il disait aussi à son amie, l'écrivaine Rachilde, en la mettant en garde contre le danger d'intoxication, car l'eau contient tous les microbes «du ciel et de la terre». C'est dans ce contexte vital pas vraiment disposé à considérer l'eau comme une boisson adéquate qu'ont surgi des écrits qui la valorisent, eux, dans sa grande et ancestrale capacité symbolique. L'œuvre d'Alfred Jarry comprend de multiples déclinaisons de l'imaginaire aquatique encore peu explorées par les critiques. Notre travail se propose d'analyser les particularités de la présence aquatique dans l'un des écrits les plus riches à cet égard, L'Autre Alceste.

Mots-clés: Alfred Jarry; L'Autre Alceste; Eau

\begin{abstract}
In one of his eccentric declarations, Alfred Jarry claimed that water is a corrosive poison, able to destroy with a single drop the purity of another liquid. He said something similar to his friend, writer Rachilde, warning her about the danger of poisoning herself, since water contains all the microbes of «sky and earth». In this vital context that appears to be utterly reluctant to consider water as a suitable beverage, arose texts which explore water in its wide and ancestral symbolic capacity. Alfred Jarry's work comprises multiple declinations of the aquatic imaginary, still little explored by critics. Our paper aims to analyze the particularities of the aquatic presence in one of Jarry's most abundant writings in this regard, L' Autre Alceste.
\end{abstract}

Keywords: Alfred Jarry; L'Autre Alceste; Water 
En una de sus excéntricas, pero habituales ocurrencias, Alfred Jarry (1873-1907) aseguraba que el agua es un veneno corrosivo, capaz de destrozar, con una sola gota, la pureza de otro líquido (por ejemplo, de la absenta, bebida adorada por el autor y, en gran medida, factor desencadenante de su prematura muerte a los 34 años de edad): «Quand ne sera-til plus besoin de rappeler que les antialcooliques sont des malades en proie à ce poison, l'eau, si dissolvant et corrosif qu'on l'a choisi entre toutes substances pour les ablutions et lessives, et qu'une goutte versée dans un liquide pur, l'absinthe par exemple, le trouble ?» (Jarry, 1987: 281). Lo mismo le decía a su amiga, la escritora Rachilde, advertiéndole sobre el peligro de envenenarse, puesto que el agua contiene todos los microbios «de la tierra y del cielo»:

\begin{abstract}
Vous vous empoisonnez, Ma-da-me, m’expliquait-il le plus sérieusement du monde. L'eau contient, en suspension, tous les microbes de la terre et du ciel, et vos sucreries, qui forment votre principale alimentation, sont des alcools à l'état rudimentaire qui saoulent bien autrement que des spiritueux convenablement expurgés par la fermentation de tous leurs principes nocifs (Rachilde, 1926: 181).
\end{abstract}

Resulta de lo anterior un contexto vital claramente reacio a considerar el agua como una bebida adecuada. Si pensamos en la preferencia de Jarry por la máscara frente a la cara, por lo artificial frente a lo natural ${ }^{1}$, vemos que hay cierta coherencia con el hecho de que lo destilado le parezca superior a aquello que se puede encontrar tal cual en la naturaleza, en este caso, el agua. Tal vez la manifestación más evidente de esta preferencia sea la ingestión ocasional de tinta china, según cuentan las anécdotas, en un gesto que opera la incorporación simbólica de lo escrito, de la literatura, por la vía alimenticia, que le correspondería de manera natural al agua.

Por otra parte, a la vez que hay este menosprecio vital por el agua, existen en la obra de Jarry escritos que la valorizan en su amplia y ancestral capacidad simbólica. Lo cual no es ninguna contradicción con lo anterior, ya que esta valorización en el arte, a servicios de la imaginación, corresponde precisamente a la conversión de lo natural en algo superior, según la estética de los simbolistas, o de los decadentes tardíos entre los que se alineaba Jarry.

La obra de Alfred Jarry comprende múltiples declinaciones del imaginario acuático, aún poco exploradas por la crítica. Este trabajo se propone analizar las particularidades de la presencia acuática en uno de sus escritos más abundantes en este aspecto, L'Autre Alceste.

L'Autre Alceste fue publicado en La Revue blanche el 15 de octubre de 1896, es decir, seis meses después de la publicación de la obra que más fama le daría a Jarry y menos de dos meses antes del escandaloso estreno de ésta. Se trata, por supuesto, de Ubu roi.

L'Autre Alceste es un texto al que su autor llama «drame en 5 récits», un sintagma problemático pues de drama realmente no hay mucho, al ser la pertenencia de esta obra al género teatral ${ }^{2}$ más bien producto del autor dixit y de que haya un elenco, una lista de personajes. Se trata de cinco narraciones retrospectivas, cinco monólogos que corresponderían a la proyección de sucesivos focos sobre el mismo plano, lográndose, a través de una poética del fragmento $^{3}$, el punto de vista múltiple que despliega la trama. En cuanto al título, el nombre podría tejer vínculos con el personaje de Misanthrope de Molière, Alceste, pero se refiere, de hecho, a Alcestis, el personaje de Eurípides. El texto de Jarry representa, entonces, una reescritura del mito griego de Alcestis. En la obra de Eurípides, ella era la mujer de Admeto, a quien las Moiras le habían concedido vivir más de lo que le tocaba si conseguía que alguien tomara su lugar, muriera por él. Y fue ella, Alcestis, la que decidió sacrificarse por su marido.

De la obra de Eurípides, poco hay en la de Jarry: básicamente, la idea de la sustitución de personas. Por lo demás, ni siquiera se vuelve a mencionar a Alcestis más allá del título. Tal como éste lo indica, se trata de una versión, es la otra Alcestis, la escrita sobre el orginal, pero sin guardar de éste más de lo necesario según la visión del nuevo artista. Y

\footnotetext{
1 «À partir de Jarry, bien plus que de Wilde, la différenciation tenue longtemps pour nécessaire entre l'art et la vie se trouve contestée, pour finir anéantie dans son principe» reza la famosa frase de André Breton (1966: 272-273).

2 Por ejemplo, St-John Perse la consideraba un poema hermético (1995).

3 Julien Schuh habla de una técnica de lo fragmentario, «dont le but essentiel est de suggérer au lecteur une unité supérieure, un centre de cohérence, voilés derrière le texte» $(2007: 10)$.
} 
Jarry decide maridar otros campos fundacionales ${ }^{4}$ para crear su obra: Admeto será ni más ni menos que el rey Salomón de los judíos, mientras que Alcestis será Balkis, o la reina de Saba, una de las esposas de Salomón. En el nuevo contexto semítico, se mantiene, sin embargo, un elemento clave de la mitología griega: el viaje al más allá a través del río, con el muerto viajando en un barco, con un barquero. Aquí, no habrá Estigia, ni Hades, y Caronte se llamará Doublemain, el ángel de la muerte.

El íncipit de L'autre Alceste enseña a Doublemain: «L'ange de la mort est apparu à mon maître avec six visages, avec lesquels il recueille les âmes des habitants de l'Orient, de l’Occident, du ciel, de la terre, des pays de Jadjudi et Madjudi et du pays des croyants. Et il a tourné vers mon maître son sixième visage» (Jarry, 19725: 909). Se trata de un fragmento críptico, para iniciados, en la vena esotérica post-simbolista de los aficionados a Swedenborg o Eliphas Levi. Un íncipit que enseña una abundancia de caras y de lugares en la enumeración y un alcance semántico de amplio espectro.

Centrándonos en el agua, aparentemente ésta falta en el íncipit. Pero está prefigurada allí. Al inicio de la obra, vemos trabajar a Salomón en un templo transparente, con parquet luminoso, que, por sus atributos de transparencia remiten al agua, y cuyas 365 puertas recuerdan la tradicional asociación del agua con el tiempo. Este número remite también a la famosa mesa, o tabla, o espejo de Salomón, «cuyos bordes y pies, en número de 365, eran de esmeralda verde»,según Ajbar Machmuâ (27), la crónica del siglo XI, mesa sobre la que se cree que el rey de los judíos escribió el verdadero nombre de Dios y que está, además, asociada al agua ${ }^{6}$.

Igualmente, al inicio de L'Autre Alceste, la caída del cuerpo terrestre del profeta se asemeja a la caída de una piedra en el agua, con la vibración del parquet luminoso.

Doublemain, el ángel de la muerte, acude a llevarse el cuerpo astral de Salomón al país de los pantanos, en un barco que navega «sur les eaux calmes qui encorbellent le paradis des croyants» (910). Destaca, en la cita anterior, lo positivo de la descripción del espacio acuático según la visión de Doublemain; las aguas son tranquilas, «paraíso», « creyentes», a lo cual se unen los atributos de levedad y fragilidad que otorga la continuación de la descripción: «flotar», «aire móvil», «peso ligero», «mi barco todavía más frágil»: «et le corps astral flottant dans l'air mobile pour venir s’asseoir à l'avant de ma barque, derrière moi, m’avertissant par son poids léger mais sur ma barque encore plus frêle, qu’il faut que je rame vers la justice d’Ankir et de Menkir» (910).

Estas características distan de hacerle eco a la configuración del más allá acuático en la mitología griega, caracterizada por la pesadez. Tampoco viene confirmada esta paz y levedad por la mención de que todo esté ocurriendo en el país de los pantanos, con el agua estancada que los caracteriza y sobre todo, con el barro, el sedimento que se opone a lo liviano infundido por el discurso de Doublemain. El pantano, mezcla de agua y tierra, no suele marcarse positivamente en su simbolismo. Bachelard habla de un imaginario de la pasta con atributos de suciedad y viscosidad ${ }^{7}$ y, para Marius Schneider, el pantano es símbolo de la descomposición del espíritu, porque faltan los dos principios activos (el aire y el fuego) y se funden en él los dos pasivos (el agua y la tierra) (Schneider 2010, apud Cirlot 300).

Esta valoración positiva del pantano por Doublemain enseña lo fundamental de este relato: la primacía de la variante, de la versión sobre lo que algunos llamarían hechos, pero que no son más que opiniones, doxa. Esta obra misma es una versión, como bien lo enseña el título. Los cinco monólogos enseñan la versión de cada personaje, sin intrusiones

\footnotetext{
4 Maurice Saillet afirma que las fuentes de L'Autre Alceste, «disparates et fort estompées», han de buscarse «dans la fable grecque ; dans le Talmud et la Kabbale, la Bible et le Coran ; peut-être aussi dans les livres sacrés de l’Égypte, de la Perse anciennes, et dans les contes profanes de l’Arabie» (1947: 31-32). Según Henri Béhar, L’Autre Alceste «déporte la mythologie grecque dans le champ sémitique de la Bible et du Coran, contant en une suite de récits rétrospectifs la tentative de substituition de l’âme de Balkis à celle de Salomon pour permettre l'achèvement du Temple» (1979: 35). Por su parte, Julien Schuh considera que, de hecho, Jarry se inspira en los escritos anónimos titulados «Légendes bibliques des Musulmans» y publicados en Le Magasin pittoresque en 1847 (2013)
}

5 En adelante, las páginas indicadas entre paréntesis sin mención del año procederán de esta referencia.

6 En el Libro de los Reyes, capítulo 7, versículos 23 a 26, abunda la referencia acuática para esta mesa: «Hizo fundir asimismo un mar de diez codos de un lado al otro, perfectamente redondo; su altura era de cinco codos, y lo ceñía alrededor un cordón de treinta codos. Y rodeaban aquel mar por debajo de su borde alrededor unas bolas como calabazas, diez en cada codo, que ceñían el mar alrededor en dos filas, las cuales habían sido fundidas cuando el mar fue fundido. Y descansaba sobre doce bueyes; tres miraban al norte, tres miraban al occidente, tres miraban al sur, y tres miraban al oriente; sobre estos se apoyaba el mar, y las ancas de ellos estaban hacia la parte de adentro. El grueso del mar era de un palmo menor, y el borde era labrado como el borde de un cáliz o de flor de lis; y cabían en él dos mil batos.»

7 Los mismos atributos son aplicables al inmundo Ubú, cuyo cetro es un cepillo de baño y en la composición corporal del cual concurre, además, el límulo; límulus es un diminutivo de limus (limo, fango). 
ajenas. Y la versión es la textualización de la visión: la dimensión visual y la repetición del verbo ver ${ }^{8}$ son abundantes en L'Autre Alceste.

En el monólogo del hijo de Salomón, Roboám, en su versión, el pantano desaparece y es sustituido por un agua caracterizada, nuevamente, por atributos positivos:

Plein de ces pensées, je suis venu vers le marais, et, comme dans les songes d'été, on court, dans un spasme douloureux ou amoureux, sur le sable sec, vers le reflux à qui le flux ne fait plus équilibre de la mer, et l'on chasse devant soi la déroute des petites vagues blanches murmurant sauve-qui-peut, je n'ai point vu le marais, mais un peu d'eau dans une prairie près d'un petit rocher entre des herbes desséchées et la lubricité au fond de cette eau du volume cylindrique des livres de mon père et de mon aieul, ébranlé sur place par les bêtes luisantes des mares, qui le soulevaient par instants, portées vers la surface par la bulle qu'ellesrespirent, et l'abandonnaient pour un peu d'air vital (914-915).

Si la descripción del pantano cambia según el punto de vista, lo que no cambia es precisamente lo que le permite a este espacio acuático ser tan cambiante: su esencia fantástica— «les intervalles fourchus des glaïeuls» (915)—y su carácter, permitiendo el desfile de matices con el cambio de la luz: «le marais, semblable à la robe d'un paon vert, à cause des myriades pressées des yeux des lentilles», según la descripción de la reina Balkis. Es un pantano en el que habitan seres extraños— «les bêtes luisantes des mares» (915), «les bêtes de l’eau», «les bêtes métalliques du marais mort» — cuyo movimiento, «la fuite dispersée des êtres de l’eau» (912), contribuye mucho al cambio constante del agua, que adquiere los destellos alucinantes de sus habitantes: «Des êtres tels que des œufs de mercure solide écrivaient et décrivaient tous les nombres et le signe de l’infini, glissant leurs éclairs sur la tôle de sable.» (911):

Aux voix et aux bruits, les œufs de mercure gyrants éclatèrent sur l'eau en déployant des ailes de viande et saignèrent dans l'air le sang des pins ; des êtres plats semblables à des pieds cornus traînant des talonnières déplumées se soulevèrent vers la face de l'eau comme les écailles de la vase. Doublemain murmura qu'il était temps qu'il plongeât ses bras jusqu'au Livre et feuilletât Hydrophilus (911-912).

Este último, Hydrophilus, remite al término que se suele usar cuando se habla de las especies de un pantano: hidrofiláceo. A la vez que, en las nuevas circunstancias, es un escarabajo y es también un libro, ya que se le puede hojear: «Et il exhuma du profond un escarbot monstrueux, couleur de poix, le ventre triangulaire vitré comme une fenêtre sur son cœur, l'établit dans la barque sur le chevalet de ses pattes, et, ouvrant à deux battants les élytres, feuilleta les ailes dépliées» (912).

El escarabajo es un coleóptero de caparazón muy fuerte, capaz de cargar 850 veces su peso, dotado con pinzas. Lo interesante es el cruce de estas características con la configuración corporal de Ubú, el personaje más famoso de Jarry, también él con caparazón y pinzas y que, además, recordémoslo, come «choux fleurs à la merdre», tal como este insecto come excrementos de animales. También viene asociado al escarabajo el barco en el que viajan Balkis y el hijo de Salomón, «le gran escarbot des marais dont la carapace était notre barque» y también Doublemain, el barquero, está asociado a él, ya que sus brazos muy largos que «se perdían en el agua lateral» (911) parecen las patas de este insecto.

Doublemain está totalmente integrado en el espacio, como si fuera un ente acuático, con las mismas características moirées de este pantano de fantasía: «Son dos m’est apparu lamé de bronze, ou couvert d'écailles très semblables à des feuilles de myrte, comme sont celles de la couleuvre» (911). El brillo de su espalda de bronce podría remitir a su doble original, Caronte, cuyo nombre deriva de charon, en griego, brillo intenso.

8 Uno de los fragmentos que enseñan la construcción del texto sobre el elemento visual (regards), revela, además, una clave del disparador creativo de Jarry: « Je détournai vers eux mes regards du rameur, et réapparurent les hommes rouges. L’un dit : « Doublemain ! que portes-tu dans ta barque rongée ? N’est-ce pas Salomon ? Quoi de plus beau que l'utile et des pots de terre superbement rangés ?» (911) Nuestro subrayado pone de manifiesto la semejanza gráfica de las tres palabras y, en el caso de las últimas dos, también fonética, lo cual podría indicar una creación del texto con base en la transformación de la palabra. 
La pertenencia acuática de Doblemano es indiscutible cuando se le enseña parecido a un pez: « Et Doublemain est venu sans marcher, les pieds unis formant la figure des deux nageoires caudales d'un poisson dressé, glissant tout droit avec le bruissement des petits cristaux du givre écrasé » (915). Doublemain es un ser del agua, a la vez que es el dueño de este espacio. Su voz surte efectos sobre la configuración acuática: «"Paix ! ou l’eau polie à ma voix va devenir boueuse et mobile, et vos pieds d'acier s’enliseront aux os de la terre.” Ayant dit, il rame» (911).

Para concluir, tenemos en L'Autre Alceste, en todos los planos, el despliegue de la versión, de lo mismo que, según la perspectiva, adquiere relieves y matices cambiantes, como Alcestis, la griega, se vuelve Belkis, la etíope, y viceversa, sin dejar de ser ellas mismas, como el agua sube y baja y se vuelve verde o azul, cambiando según las circunstancias y manteniendo siempre su esencia formada por hidrógeno y oxígeno. Es, en definitiva, otra ilustración de la patafísica, «la plus haute tentation de l'esprit» según Baudrillard (2002: 15), para la cual el reloj redondo es cuadrado, «elliptique de trois quarts» (Jarry, 1972: 669) y una cosa es lo que es y, sobre todo, su contrario.

\section{Referencias bibliográficas}

Ajbar Machmuâ, Crónica anónima del siglo XI (1867). Ed. y trad. E. Lafuente y Alcantara. 2 vols. Madrid. $<$ http://www.bibliotecavirtualdeandalucia.es/catalogo/consulta/registro.cmd?id=100088 $>$ [Consultado el 14 de junio 2016].

BACHELARD, Gaston (2005). El agua y los sueños: ensayo sobre la imaginación de la materia. Madrid: Fondo de Cultura Económica. BAUDRILLARD, Jean (2002). Pataphysique. Paris: Sens \&Tonka.

BÉHAR, Henri (1979). «La culture potachique à l'assaut du symbolisme: le cas Jarry» en L'Étoile-Absinthe, Octobre 1979, p. $32-43$.

Breton, André (1966). Anthologie de l'humour noir. Paris: Jean-Jacques Pauvert.

CiRLOT, Juan Eduardo (2004). Diccionario de símbolos. Madrid: Siruela.

EURÍPIDES (1972). Alcestis. Las bacantes. El cíclope. Madrid: Espasa-Calpe.

JARrY, Alfred (1972). L'Autre Alceste, dans Euvres complètes I. Paris: Gallimard, p. 907-916.

JARRY, Alfred (1987). Euvres complètes II. Paris: Gallimard.

RACHILDE (1926). Alfred Jarry ou le surmâle de lettres. Paris: Grasset.

SAILlet, Maurice (1947). «Commentaire» dans Alfred Jarry, L’Autre Alceste, Paris: Fontaine.

SCHUH, Julien (2010). «Jarry et Le Magasin pittoresque : une érudition familière» en L'Etoile-Absinthe n ${ }^{\circ}$ 123-124, p.101-134.

Schun, Julien (2007). «Marcel Schwob et Alfred Jarry: Des difficultés de la synthèse». En Colloque international: D’un siècle à l'autre: retour à Marcel Schwob et à Claude Cahun. Presses Universitaires de Rennes, p. 113-125.

SCHNEIDER, Marius (1998). El origen musical de los animales-símbolos en al mitología y la escultura antiguas. Madrid: Siruela.

PERSE, Saint-John (1995). «Correspondance inédite (1936-1961)» en Europe, n 799-800, p. 65-103. 